\title{
FOI BOM PARA VOCÊ? UMA COMPARAÇÃO DO VALOR HEDÔNICO DE COMPRAS FEITAS EM DIFERENTES TIPOS DE VAREJISTAS
}

HOW DID YOU LIKE IT? COMPARING HEDONIC SHOPPING VALUE OF PURCHASES MADE AT DIFFERENT TYPES OF RETAILERS

\section{JOSÉ MAURO DA COSTA HERNANDEZ}

Livre-docente pela Universidade de São Paulo (USP). Professor do Programa de Pós-Graduação em Administração da Universidade Nove de Julho (Uninove). Avenida Francisco Matarazzo, 612, $2^{\circ}$ andar, Barra Funda São Paulo - SP - CEP 05001-100 E-mail: jmhernandez@uninove.br 


\section{RESUMO}

Este estudo compara, por meio de três pesquisas distintas, o valor hedônico de compras feitas em diferentes tipos de varejistas. Na primeira pesquisa, comparouse o valor hedônico de compras feitas em lojas eletrônicas e lojas tradicionais; na segunda, foram comparados os valores hedônicos de compras feitas em shopping centers de diferentes tamanhos; e na última comparou-se o valor hedônico de compras feitas em lojas de especialidade e superlojas. De forma geral, a conclusão é que o valor hedônico não pode ser atribuído a classes específicas de varejistas, uma vez que os efeitos principais, quando presentes, foram pouco significativos. Sugere-se que futuros estudos concentrem-se em determinadas características dos varejistas e na interação destas características com características individuais na criação do valor hedônico.

\section{PALAVRAS-CHAVE}

Marketing; Comportamento do consumidor; Valor hedônico de compras; Varejo; Levantamento.

\section{ABSTRACT}

This study compares, in three distinct studies, the hedonic value of purchases made at different retail merchants. In the first study it is compared the hedonic shopping value of purchases made at e-stores and brick-and-mortar merchants; the second compares large and small malls, and the third compares specialty stores and superstores. In general, the conclusion is that hedonic shopping value cannot be attributed to specific merchant classes since the main effects, whenever present, were of little significance. It is suggested that future studies should focus on looking into specific retail merchant characteristics and how they interact with individual characteristics in the creation of hedonic value. 


\section{KEYWORDS}

Marketing; Consumer behavior; Hedonic shopping value; Retailing; Survey.

\section{INTRODUÇÃO}

À medida que surgem novos formatos de varejo, a concentração dos negócios em torno de poucos varejistas aumenta, o comércio eletrônico cresce a taxas surpreendentes, e também cresce a pressão competitiva no mercado varejista. Nesse ambiente cada vez mais instável, a forma tradicional de fazer negócios, isto é, atrair os consumidores com maiores sortimentos, menores preços, mais atividade promocional e horários de atendimento mais elásticos, já não é mais suficiente (ARNOLD; REYNOLDS, 2003).

Os varejistas têm reagido de formas diferentes a essas mudanças ambientais. Enquanto alguns continuam a insistir nas mesmas velhas estratégias de sempre, outros buscam melhorar a qualidade do serviço e investem em treinamento de pessoal. Outros ainda tentam tornar as suas lojas um lugar mais divertido e alegre para fazer compras, oferecendo maior valor hedônico de compras aos seus clientes (BURKE, I997).

Cada vez mais se reconhece a importância do entretenimento como uma ferramenta estratégica para se competir no mercado varejista. Nos Estados Unidos, a tendência de combinar entretenimento e vendas a varejo já se tornou tão frequente que até uma nova palavra em inglês foi cunhada para referir-se a esse fenômeno: retailtainment.

Novos shopping centers já são planejados para acomodar parques de diversão, complexos gigantescos de cinemas e áreas de alimentação com restaurantes que satisfazem todos os tipos de paladar. A ideia desses novos empreendimentos é atrair mais consumidores ao oferecer conveniência e entretenimento sob o mesmo teto. Hoje, as lojas já não são mais criadas em torno de produtos, mas sim em torno de conceitos. A ideia é apelar para um número cada vez maior de sentidos humanos.

Atualmente, ter um grande sortimento prontamente disponível é o nível mínimo necessário para competir. Proporcionar uma compra utilitária é necessário, mas já não é mais suficiente para competir com sucesso. Cada vez mais os consumidores desejam satisfazer as suas necessidades de compra da forma mais hedônica possível.

Os primeiros estudos sobre a dimensão hedônica do consumo foram publicados no início dos anos I980 (HIRSCHMAN; HOLBROOK, I982; HOLBROOK; 
HIRSCHMAN, I982). Enquanto a abordagem tradicional mantém seu foco principalmente sobre os aspectos utilitários e funcionais do consumo, a dimensão hedônica concentra-se nos aspectos subjetivos, simbólicos, emotivos, inconscientes e psicológicos.

Uma das principais linhas de pesquisa nessa área de estudos investiga os valores hedônicos e utilitários da compra (SHERRY JR., I990; BABIN; DARDEN; GRIFFIN, I994; BABIN; DARDEN, I995; WAKEFIELD; BARNES, I996; CHILDERS; CARR; PECK, 200I; ARNOLD; REYNOLDS, 2003; BARDHI; ARNOULD, 2005; JONES; REYNOLDS; ARNOLD, 2006). O valor hedônico da compra tem recebido tal atenção por já se ter demonstrado que ele está relacionado a diversas variáveis de interesse para o varejo, como a lealdade do consumidor, a satisfação com o varejista, tempo e dinheiro gastos numa loja, intenção de comprar por impulso, intenção de voltar a comprar de um varejista e o boca a boca positivo.

Em geral, os estudos anteriores nessa área concentraram-se principalmente na identificação dos antecedentes dos valores hedônico e utilitário da compra (DONOVAN et al., I994; DONOVAN; ROSSITER, I994; BABIN; DARDEN, I995; WAKEFIELD; BAKER, I998; STOEL; WICKLIFFE; LEE, 2004) e, menos frequentemente, em seus consequentes (BABIN; DARDEN, I996; BABIN; ATTAWAY, 2000; BABIN et al., 2005).

Poucos estudos, entretanto, se interessaram em comparar o valor hedônico que diferentes tipos de varejistas proporcionam aos consumidores. Portanto, o objetivo deste artigo é preencher parcialmente essa lacuna ao comparar o valor hedônico de compras feitas em diferentes tipos de varejistas por meio de três pesquisas distintas. Na primeira delas, compara-se o valor hedônico de compras feitas em lojas eletrônicas e lojas tradicionais; na segunda pesquisa, compara-se o valor hedônico de compras feitas em shopping centers de diferentes tamanhos; e, finalmente, na terceira compara-se o valor hedônico de compras feitas em lojas de especialidade e superlojas.

Uma vez que é crescente o número de varejistas que se esforça em oferecer mais entretenimento aos seus consumidores como forma de mantê-los felizes e satisfeitos, é importante investigar se eles estão tendo sucesso nessa empreitada. Com base nesse tipo de abordagem, talvez seja possível aprender com as experiências dos varejistas que tenham obtido sucesso em seus esforços, a fim de que se possam desenvolver práticas gerenciais mais eficientes.

Com essas questões em vista, o presente artigo, além desta introdução, foi assim dividido: inicialmente, são revistos os conceitos de valor hedônico e utilitário de compras, quando também se formulam as hipóteses que guiaram as três pesquisas empíricas; a seguir, apresentam-se o método utilizado na coleta e análise dos dados das três pesquisas e os resultados obtidos; e, finalmente, discutem-se os resultados e apresenta-se a conclusão deste artigo. 


\section{REVISÃO DA LITERATURA}

\subsection{VALOR HEDÔNICO DE COMPRA VERSUS VALOR UTILITÁRIO DE COMPRA}

Quando surgiu a necessidade de uma investigação mais aprofundada sobre os aspectos intangíveis e subjetivos do consumo, Hirschman e Holbrook (I982) se colocaram entre os primeiros autores a reconhecer o fato de que a experiência de compras pode ser intrinsicamente satisfatória. Para Hirschman e Holbrook (I982, p. 92), os aspectos intangíveis e subjetivos do consumo, ao contrário dos tangíveis e funcionais, pertencem à esfera do consumo hedônico, "um fenômeno que designa as facetas do comportamento do consumidor relacionadas aos aspectos multissensoriais e emocionais da experiência de um indivíduo com os produtos".

A abordagem experimental proposta por Hirschman e Holbrook (I982) refere-se aos aspectos simbólicos, emocionais, insconscientes, estéticos e psicológicos do consumo, enquanto a abordagem tradicional do comportamento do consumidor lida com os aspectos objetivos, tangíveis, cognitivos, conscientes, utilitários e econômicos.

Três principais linhas de pesquisas têm se concentrado em investigar as facetas hedônicas e utilitárias do consumo. A primeira concentra-se nas características dos consumidores: consumidores hedônicos são aqueles que buscam estímulos sensoriais e inconscientes em suas experiências de compra e consumo de produtos, enquanto consumidores utilitários se comportam mais racionalmente e se preocupam com a funcionalidade dos bens (BELLENGER; KORGAONKAR, I980; BLOCH; RIDGWAY; NELSON, I99I).

A segunda linha de pesquisa concentra-se nas características de produtos e marcas (BATRA; AHTOLA, I990; MANO; OLIVER, I994; DHAR; WERTENBROCH, 2000; PARK; MOON, 2003; VOSS; SPANGENBERG; GROHMANN, 2003) e propõe que, assim como os consumidores, produtos e marcas podem ser predominantemente hedônicos por proporcionarem mais prazer, emoção e alegria àqueles que os compram, como roupas, carros esportivos e relógios de luxo, ou predominantemente utilitários por terem principalmente características instrumentais e utilitárias, como os computadores pessoais, minivans e fornos de micro-ondas.

A terceira linha de pesquisa, na qual este estudo se encaixa, concentra-se no valor derivado de uma compra (SHERRY JR., I990; BABIN; DARDEN; GRIFFIN, I994; BABIN; DARDEN, I995; WAKEFIELD; BARNES, I996; CHILDERS; CARR; PECK, 200I; ARNOLD; REYNOLDS, 2003; BARDHI; ARNOULD, 2005; JONES; REYNOLDS; ARNOLD, 2006). O conceito de valor refere-se àquilo que 
os consumidores percebem que têm a ganhar com a experiência de compra (HOLBROOK, I986). Por sua vez, segundo Babin, Darden e Griffin (I994), uma visão mais ampla do conceito de valor reconhece tanto uma dimensão utilitária, resultado de um esforço consciente para atingir uma consequência desejada, quanto uma dimensão hedônica, que procura capturar a dualidade básica das recompensas do comportamento humano. Essas duas dimensões refletem a distinção entre fazer algo simplesmente para obter algo e fazer algo simplesmente pelo prazer de fazê-lo.

O valor utilitário da compra foi descrito como racional (BATRA; AHTOLA, I990) e associado a trabalho duro (FISCHER; ARNOLD, I990), resultado da aquisição eficiente e deliberada de um produto (BABIN; DARDEN; GRIFFIN, I994). O valor utilitário está associado à eficiência da compra e à correta escolha do produto com base na avaliação lógica das informações disponíveis sobre o produto. Não é necessário que a compra tenha sido concluída para que a experiência gere valor utilitário, uma vez que os consumidores podem simplesmente se engajar na coleta de informações para aquisições futuras.

Por sua vez, o valor hedônico da compra reconhece o lado festivo, lúdico e epicurista da compra (SHERRY JR., I990). O valor hedônico é mais subjetivo e pessoal, dirigindo-se para a satisfação de desejos individuais específicos (HOLBROOK; HIRSCHMAN, I982). O valor hedônico é um fim em si mesmo e, portanto, não necessariamente está associado a uma compra. A eficiência não é uma preocupação, assim como não se sabe onde a compra começa nem onde termina.

Atualmente, parece estar bem estabelecido que uma compra pode oferecer os dois tipos de valores (JONES; REYNOLDS; ARNOLD, 2006). Por exemplo, Bardhi e Arnould (2005) descobriram que até mesmo a procura por uma barganha pode se tornar uma experiência hedônica.

Nos últimos dez anos aproximadamente, as pesquisas nessa área têm se concentrado extensivamente na identificação dos antecedentes dos valores hedônicos e utilitários da compra (BABIN; DARDEN, I995; DONOVAN; ROSSITER, I994; DONOVAN et al., I994; STOEL; WICKLIFFE; LEE, 2004; WAKEFIELD; BAKER, I998) e até certo ponto em seus resultados (BABIN; ATTAWAY, 2000; BABIN; DARDEN, I996; BABIN et al., 2005).

Em geral, estudos anteriores descobriram que o valor hedônico está fortemente associado à atmosfera da loja (DONOVAN et al., I994; MATTILA; WIRTZ, 200I). Fatores como o sortimento da loja, os preços, a equipe de vendas, o fato de a loja estar ou não muito cheia e outros elementos periféricos dos serviços do varejista também contribuem para a natureza de entretenimento da compra (COTTET; LICHTLÉ; PLICHON, 2006; JONES, I999). 
Em relação aos consequentes, já se descobriu que o valor hedônico da compra contribui para aumentar o afeto dos consumidores pela loja (MANO; OLIVER, I994) e a intenção de voltar para fazer compras nela (STOEL; WICKILIFFE; LEE, 2004). Também já se demonstrou que o valor hedônico da compra está positivamente associado à satisfação do consumidor com o varejista (BABIN et al., 2005; COTTET; LICHTLÉ; PLICHON, 2006), à intenção de comprar por impulso (MATTILA; WIRTZ, 200I), ao tempo gasto na loja (DONOVAN et al., I994; SHERMAN; MATHUR; SMITH, I997), à lealdade do consumidor e ao boca a boca positivo (JONES; REYNOLDS; ARNOLD, 2006).

\subsection{VALOR HEDÔNICO DE COMPRAS EM LOJAS ELETRÔNICAS E TRADICIONAIS}

Pesquisas anteriores demonstraram que o valor hedônico da compra resulta particularmente de elementos da atmosfera da loja, como decoração, vitrines, expositores, iluminação, cores, músicas e odores. Uma loja tradicional, isto é, a loja física de tijolo e cimento, representa uma infinitude de estímulos sensoriais para todos os sentidos do corpo humano: olfato, paladar, audição, visão e tato. Um conjunto considerável de literatura já foi produzido sobre os efeitos positivos da amosfera das lojas tradicionais (PAN; ZINKHAN, 2006). Entretanto, o impacto desses fatores em ambientes virtuais ainda não está bem documentado (EROGLU; MACHLEIT; DAVIS, 2003).

Uma vez que uma loja eletrônica proporciona apenas estímulos visuais e auditivos, é de supor que ela esteja em séria desvantagem em comparação às suas concorrentes tradicionais em termos de valor hedônico. Entretanto, alguns estudos argumentam que, embora os atributos utilitários e instrumentais das lojas eletrônicas, como a facilidade e a conveniência da compra, sejam os fatores determinantes das atitudes e dos comportamentos dos consumidores, características hedônicas específicas do meio eletrônico poderiam exercer um papel tão importante quanto os aspectos utilitários ao conformar esses comportamentos (CHILDERS; CARR; PECK, 200I).

De fato, estudos anteriores demonstraram que o valor hedônico também pode ser derivado dos ambientes eletrônicos de compra (CHILDERS; CARR; PECK, 200I; JARVENPAA; TODD, I997) e impactar positivamente as atitudes em relação às lojas eletrônicas. Por exemplo, Fiore, Jin e Kin (2005) descobriram que o prazer resultante de uma imagem interativa de um website estimula tanto a atitude global quanto a vontade de comprar em uma loja eletrônica, enquanto Eroglu, Machleit e Davis (2003) constataram que a qualidade atmosférica de uma loja eletrônica aumenta o nível de prazer que os compradores sentem, o que, por sua vez, influencia a sua atitude e a satisfação com a loja eletrônica. Fiore, Lee e 
Kunz (2004) sugerem que a novidade de uma nova tecnologia como o comércio eletrônico poderia resultar em maior prazer e excitação.

O interesse deste estudo é comparar o valor hedônico de compras feitas em lojas eletrônicas e tradicionais. Embora exista uma infinidade de recursos de interatividade que uma loja eletrônica possa implementar para estimular os sentidos humanos, as limitações da velocidade de transmissão de dados e dos recursos tecnológicos dos consumidores ainda representam barreiras reais para as lojas eletrônicas poderem implementar esses recursos. Portanto, por causa da maior capacidade de estimular os sentidos humanos, as lojas tradicionais são capazes de oferecer maior valor hedônico que as eletrônicas, o que permite a formulação da seguinte hipótese:

- Hi: As compras feitas em lojas tradicionais oferecem maior valor hedônico que as compras feitas em lojas eletrônicas.

\subsection{VALOR HEDÔNICO DE COMPRAS EM GRANDES E PEQUENOS SHOPPING CENTERS}

Os shopping centers já se tornaram os templos modernos do consumo em praticamente todos os lugares do mundo, e a pressão competitiva nesse segmento nunca foi tão grande. A fim de diferenciarem-se dos competidores e atrair um número crescente de consumidores, os shopping centers têm procurado aumentar a diversidade de suas lojas. Eles tentam atrair um número maior de lojas com marcas bem conhecidas para convencer os consumidores que a visita vale a pena, pois lá eles encontrarão as marcas que procuram. As grandes cadeias de lojas de marcas reconhecidas tomaram o lugar antes ocupado pelas grandes lojas de departamento. Além disso, os shopping centers tentam proporcionar mais conveniência para aqueles que preferem comprar tudo num lugar só ao atrairem lojas como supermercados, bancos, farmácias, postos de gasolina e mecânicas para manutenção de veículos. Como toque final, tentam equilibrar a área de compras com áreas cada vez mais maiores de lazer, que incluem parques de diversão, cinemas e restaurantes que atendem a todos os tipos de paladares.

A tendência de combinar entretenimento com varejo já se tornou tão prevalente nos Estados Unidos que até mesmo uma nova palavra em inglês já foi cunhada para referir-se a esses empreendimentos: retailtainment. A ideia por trás desse movimento é que quanto mais favoráveis forem as percepções dos consumidores sobre os atributos de um shopping center, maior será o seu desejo de frequentá-lo para preencher as suas necessidades de consumo e diversão (SHIM; EASTLICK, I998). 
Existem, entretanto, evidências de que o entretenimento excessivo pode distrair os consumidores e levá-los para longe das compras. Por exemplo, Christiansen et al. (I999) descobriram que o nível de entretenimento que um shopping center oferece está negativamente relacionado ao número de itens comprados.

Pesquisas anteriores proporcionaram evidências empíricas de que a escolha de um shopping center por um consumidor é influenciada pela sua variedade de lojas (BELLENGER; ROBERTSON; GREENBERG, I977), pela sua imagem (FINN; LOUVIERE, I996) e pela frequência com que os clientes o visitam (STOLTMAN; GENTRY; ANGLIN, I99I). Também se demonstrou que a variedade de lojas de um shopping center está relacionada à sensação estimulante de estar em um shopping center (KOWINSKI, I985; WAKEFIELD; BAKER, I998). É muito provável que os consumidores que queiram aproveitar ao máximo o tempo dedicado às compras não hesitarão em trocar shopping centers menores e mais próximos por aqueles maiores e mais distantes, mas que ofereçam uma maior variedade de lojas e opções de entretenimento.

Portanto, quanto maior o número e a variedade de lojas que um shopping oferece, maior deve ser a sua capacidade de proporcionar maior valor hedônico aos seus clientes, o que conduz à seguinte hipótese:

- H2: $\mathrm{O}$ valor hedônico de uma compra está associado ao número e à variedade de lojas do shopping center.

\subsection{VALOR HEDÔNICO DE COMPRAS FEITAS EM LOJAS DE ESPECIALIDADE E SUPERLOJAS}

Em geral, as lojas de especialidade são pequenas, mantêm em estoque um grande número de itens de algumas poucas linhas de produtos e proporcionam nível de serviço de alta qualidade aos seus clientes por meio de uma equipe de vendas bem treinada (KOTLER; ARMSTRONG, 200I). Lojas de roupas, CD, móveis e livros são bons exemplos desses tipos de lojas. As superlojas, por sua vez, são maiores que os supermercados e mantêm em suas prateleiras um pequeno número de itens de uma diversidade enorme de linhas de produtos (KOTLER; ARMSTRONG, 200I). As superlojas também costumam proporcionar ao cliente a possibilidade de frequentar outros tipos de lojas e serviços como farmácias, caixas automáticos, óticas, relojoarias, lavanderia, revelação de fotos e até mesmo serviços para animais de estimação. No Brasil, são bons exemplos desse tipo de loja o Carrefour, o Extra, o Wal-Mart e algumas unidades das Lojas Americanas.

Em comparação com uma superloja, uma loja de especialidade oferece muito mais oportunidades para os consumidores encontrarem itens específicos 
que nem sempre estão disponíveis em lojas que vendem muitas linhas de produtos. Esse tipo de loja também proporciona aos consumidores mais possibilidades para explorar novos itens e obter informações sobre os produtos desejados. Essas lojas geralmente possuem mais vendedores e muito bem treinados sobre os produtos que comercializam para atender os clientes.

As lojas de especialidade são cada vez menos construídas em torno de produtos e cada vez mais em torno de conceitos. Em diversos lugares do mundo, já existem lojas em que o cliente pode jogar basquete dentro do estabelecimento para experimentar o desempenho do tênis, atirar com uma arma de caça para sentir o seu "coice" e até mesmo praticar alpinismo com o equipamento desejado. O número de restaurantes temáticos é ainda maior: Planet Hollywood (estrelas de Hollywood), Hard Rock Cafe (rock) e Rainforest Cafe (floresta tropical) são alguns dos mais conhecidos exemplos americanos. Estes são bons exemplos de varejistas "experienciais", um tipo de varejista que está preocupado em oferecer aos seus clientes uma experiência de compra que vai muito além dos itens adquiridos.

A atmosfera cuidadosamente preparada das lojas de especialidade as torna mais sofisticadas, confortáveis e intimistas. Essas lojas também têm a capacidade de proporcionar serviços mais personalizados do que as superlojas e geralmente estão localizadas em locais de fácil acesso como shopping centers ou áreas comerciais tradicionais.

As superlojas, por sua vez, oferecem aos consumidores a conveniência de encontrar um vasto sortimento de produtos e serviços diferentes no mesmo lugar. A decoração é simples e, em geral, pouco atraente. O serviço é padronizado, mas eficiente. As superlojas geralmente estão localizadas em regiões mais distantes do centro da cidade onde os terrenos são mais baratos, embora não seja difícil encontrar uma vaga em seus grandes estacionamentos.

Uma vez que a atmosfera da loja é um dos principais determinantes do valor hedônico de compras (DONOVAN et al., I994; MATTILA; WIRTZ, 200I) e tendo em mente que as lojas de especialidade oferecem maiores possibilidades de obter informações sobre os produtos e de entreter-se com o vasto sortimento que elas oferecem, por hipótese as compras feitas nesse tipo de loja oferecem maior valor hedônico que as compras feitas em superlojas.

- $\quad \mathrm{H}_{3}$ : As compras feitas em lojas de especialidade oferecem mais valor hedônico que as compras realizadas em superlojas. 


\section{MÉTODO}

Para testar as hipóteses apresentadas na seção anterior, 583 pessoas foram entrevistadas em três pesquisas diferentes conduzidas em uma das maiores cidades brasileiras. As entrevistas foram feitas por alunos de graduação apropriadamente treinados sobre os objetivos de cada um dos estudos. Os entrevistados foram abordados em áreas próximas a shopping centers e em ruas com grande concentração de lojas.

A estrutura do questionário dos três estudos era basicamente a mesma. A primeira parte continha algumas questões-filtro para selecionar os respondentes de acordo com o objetivo da pesquisa. A segunda apresentava questões relacionadas a uma determinada compra (o que foi comprado e quando, quanto foi pago, quanto tempo levou a compra e quais as razões para ter feito a compra naquela loja específica). A terceira parte continha os II itens da escala de valor de compra pessoal (ver Quadro I), e a última, questões sobre dados demográficos.

Para mensurar o valor hedônico de compras, deu-se preferência à subescala de valor hedônico da escala de valor de compra pessoal (VCP) desenvolvida por Babin, Darden e Griffin (I994), embora existam outras mais recentes (ARNOLD; REYNOLDS, 2003). A escala VCP tenta capturar tanto o valor hedônico quanto o utilitário de uma compra, e a sua escolha se deu pelo fato de ela ter sido validada várias vezes (JONES; REYNOLDS; ARNOLD, 2006) e por conter menos itens que outras escalas.

Todos os itens da subescala de valor hedônico de compras (VHC) foram traduzidos para a língua portuguesa pelo autor deste estudo e submetidos à análise de colegas especialistas em varejo. Depois de algumas pequenas mudanças, a escala foi submetida a um pré-teste entre pessoas com características semelhantes às das pessoas que seriam entrevistadas nas pesquisas subsequentes. Esse pré-teste demonstrou a necessidade de ajuste de alguns itens. Especificamente, os termos escape e excitement of the hunt, presentes, respectivamente, nos itens 3 e 8 da escala original, quando traduzidos para a língua portuguesa, não refletem expressões usualmente utilizadas por brasileiros para se referir às atividades de compras.

Esses dois itens foram então modificados para "distração" e "grande prazer". A tradução dos demais itens ficou bastante próxima à forma original (Quadro I). Um novo pré-teste foi realizado, e não se observou nenhum problema. Assim como na escala original, as respostas a cada item foram indicadas numa escala do tipo Likert com 5 pontos. 


\section{QUADRO I}

\section{ITENS DA ESCALA DE VALOR HEDÔNICO DE COMPRAS}

ITENS DA ESCALA

1 Eu me diverti fazendo esta compra.

2 Eu fiz esta compra porque quis, não foi por obrigação.

3 Fazer esta compra foi uma verdadeira distração para mim.

4 Comparando com outras coisas que eu poderia ter feito, o tempo gasto nesta compra foi muito mais agradável.

5 Eu gostei de ficar olhando os novos produtos.

6 Eu gostei da compra em si, não apenas pelo produto que comprei.

7 Eu me diverti fazendo esta compra porque eu pude fazê-la sem pressa.

8 A busca por esse produto me deu grande prazer.

9 Esqueci meus problemas enquanto fazia esta compra.

10 Senti um certo espírito de aventura enquanto fazia esta compra.

11 Esta compra foi um jeito muito legal de passar o tempo.

Fonte: Adaptado de Babin, Darden e Griffin (I994).

Considerando a importância da adaptação da escala VHC para os resultados do estudo, alguns testes foram feitos para analisar a validade e a confiabilidade da escala adaptada. A confiabilidade de construto foi avaliada pela sua consistência interna pelo cálculo do coeficiente de alfa de Cronbach (DEVELLIS, 2003). Os coeficientes variaram entre 0,795 e 0,837 nas três pesquisas, acima do limite inferior sugerido por Nunnally e Bernstein (I994). Quando os dados dos três estudos foram consolidados, o coeficiente resultante foi 0,876 .

A validade de critério (DEVELLIS, 2003) foi avaliada por meio do cálculo da correlação de Pearson entre a escala VHC e outras duas medidas: valor e tempo gastos na compra do produto. Teoricamente, quanto maior o valor hedônico de uma compra, maior o tempo e o valor gastos na compra (BABIN; DARDEN; GRIFFIN, I994). De fato, verificou-se uma correlação positiva e significativa entre a escala VHC adaptada e o valor pago pelo produto comprado $(\mathrm{r}=0,353, \mathrm{p}<\mathrm{0}, \mathrm{OI})$, e entre a escala VHC adaptada e o tempo gasto na compra $(r=0,224, p<0,0 I)$.

A validade preditiva da escala VHC adaptada também foi avaliada. Para tanto, verificou-se a capacidade da escala adaptada de prever o valor hedônico de 
diferentes categorias de produtos. Diversos estudos classificaram roupas como uma categoria de produtos com alto valor hedônico (VOSS; SPANGENBERG; GROHMANN, 2003). As roupas estão associadas à moda, que, por sua natureza, em constante mudança, cria uma ilusão de algo novo, fantástico, ilusório, o que acaba tornando as roupas mais sujeitas ao consumo hedônico. Portanto, a compra de roupas (e acessórios como sapatos e bolsas) deveria proporcionar maior valor hedônico do que a compra de produtos como CD, DVD e livros, itens que não estão associados a características hedônicas.

Os dados dos três estudos foram combinados, e os valores médios da escala VHC foram comparados por meio de um teste ANOVA. Compararam-se nesse teste apenas as compras de cinco categorias de produtos (roupas, sapatos, bolsas, CD, DVD e livros), que juntas representavam 92\% das respostas válidas das três pesquisas.

A hipótese de que os valores médios da escala VHC para as cinco categorias de produtos eram iguais foi rejeitada $(\mathrm{F}(4,540)=\mathrm{IOI}, 29, \mathrm{p}<0,00 \mathrm{I})$. Testes post hoc de Scheffé revelaram que, no nível de confiança de $5 \%$, o valor hedônico da compra de roupas $(M=3,82)$ e sapatos e bolsas $(M=3,78)$ é significativamente maior que o valor hedônico da compra de CD $(M=2,55)$, DVD $(\mathrm{M}=2,55)$ e livros $(\mathrm{M}=2,48)$.

Em resumo, esses resultados proporcionam fortes evidências de que a escala VHC adaptada é válida e confiável.

\section{RESULTADOS}

A fim de testar a hipótese de que lojas tradicionais proporcionam maior valor hedônico de compras do que as eletrônicas, uma amostra conveniente de I87 indivíduos foi entrevistada. Para evitar o viés causado pela análise de diferentes categorias de produtos, a amostra incluiu apenas indivíduos que haviam comprado categorias de produtos similares em termos de características hedônicas. Foram escolhidas três categorias de produtos: CD, DVD e livros. Optou-se por esses produtos porque ainda são as categorias mais vendidas pela internet (EBIT, 2006), o que tornaria mais fácil o recrutamento de respondentes para a pesquisa. Para que os entrevistados pudessem responder às perguntas com precisão, a pesquisa foi realizada apenas com pessoas que haviam feito a compra nos últimos I5 dias.

Das pessoas entrevistadas, $44 \%$ eram homens, $64 \%$ tinham 30 anos ou menos de idade, $33 \%$ tinham curso universitário completo e $37 \%$ tinham renda mensal superior a $\mathrm{R} \$ 5.000,00$. Em relação à categoria de produtos, $44 \%$ decla- 
raram a compra de livros, $34 \%$ de CD e $22 \%$ de DVD. Em média, as pessoas gastaram $\mathrm{R} \$ 35,00$ e levaram cerca de 20 minutos para fazer suas compras; realizaram-se cerca de dois terços $(6 \mathrm{I} \%)$ dessas compras em lojas tradicionais.

Para testar a hipótese de que o valor hedônico de compras feitas em lojas tradicionais é maior que as realizadas em lojas eletrônicas, as médias da escala VHC adaptada para os dois grupos foram comparadas por meio de um test t. Ao contrário do que era esperado, o valor hedônico das compras feitas em lojas eletrônicas $(\mathrm{M}=2,54)$ foi superior ao valor hedônico das compras realizadas em lojas tradicionais $(M=2,39)$, embora o resultado seja significativo apenas no nível de ı०\% (t(I85) =-I,87). Portanto, Hi deve ser rejeitada.

Para testar a segunda hipótese, 202 indivíduos que haviam acabado de fazer uma compra em um de seis shopping centers convenientemente selecionados foram entrevistados. Os shopping centers foram selecionados em razão do número de lojas que cada um possui como forma de aumentar a diversidade de tamanhos de shopping centers na amostra. Mais especificamente, o maior shopping center tinha 504 lojas na época da pesquisa, e o menor, apenas 30 lojas; os demais tinham I3I, 202, 203 e 37I lojas.

Das pessoas entrevistadas, $44 \%$ eram homens, $51 \%$ tinham mais de 30 anos de idade, $35 \%$ tinham concluído o curso superior e i6\% tinham renda superior a $\mathrm{R} \$ 5.000,00$. O item mais comprado pelos respondentes foi roupas $(52 \%)$, seguido por sapatos e bolsas (i6\%). As demais compras, representando $32 \%$ do total, estavam distribuídas em i 6 categorias diferentes de produtos. Em média, as pessoas gastaram cerca de $\mathrm{R} \$$ IIO,, 0 e levaram aproximadamente 48 minutos para fazer suas compras. O número de entrevistas realizadas em cada shopping center foi muito próximo.

Cada respondente foi apresentado a duas frases relacionadas ao número e à variedade de lojas do shopping center, devendo indicar, numa escala de cinco pontos do tipo Likert, o quanto ele concordava com cada frase ou discordava dela. Para testar a hipótese de que o número e a variedade de lojas de um shopping center estão associados ao valor hedônico das compras feitas ali, estas duas escalas foram correlacionadas com a escala VHC adaptada. A correlação de Pearson entre o tamanho percebido do shopping center e o valor hedônico de compras, medida pela escala VHC adaptada, foi igual a o,I43 ( $\mathrm{p}<\mathrm{o,IO})$, enquanto a correlação de Pearson entre a variedade de lojas percebida do shopping center e o valor hedônico de compras foi igual a o,I2I, resultado não estatisticamente significativo.

Considerando que as duas medidas anteriores eram medidas de percepção, um teste adicional foi feito para comparar o valor hedônico proporcionado por cada shopping center. Uma vez que o número de lojas de cada shopping era diferente, seria esperado que aqueles com mais lojas proporcionassem maior valor hedônico que os com menos lojas. 
Assim, a amostra foi dividida em seis subamostras, cada uma delas correspondendo a um dos shopping centers, e verificou-se por meio de um teste ANOVA se havia diferença significativa entre as médias da escala VHC adaptada. Contrário ao que era esperado, não foram encontradas diferenças significativas entre as médias da escala VHC para cada shopping center $(\mathrm{F}(5, \mathrm{I} 96)=0,85$, n.s. $)$. Portanto, H2 também deve ser rejeitada.

Para testar a terceira hipótese, uma amostra conveniente de 194 indivíduos foi entrevistada. A amostra incluiu apenas pessoas que tinham acabado de fazer a compra de CD, DVD e livros. Novamente, a razão pela escolha desses itens é que eles são similares em termos de características hedônicas, tornando a comparação menos enviesada.

Dentre os entrevistados, $48 \%$ eram homens, $73 \%$ tinham menos de 30 anos de idade, $22 \%$ tinham terminado o curso superior e Io\% tinham renda superior a $\mathrm{R} \$ 5.000,00$. Livros (47\%) foi a categoria mais comprada pelos respondentes, seguida por CD (31\%) e DVD (22\%). Em média, as pessoas gastaram R 30,00 em suas compras que levou, em média, 27 minutos. Aproximadamente três quartos $(77 \%)$ dos entrevistados tinham feito suas compras em lojas de especialidade, e os demais 33\% em superlojas.

Para testar a hipótese de que as compras feitas em lojas de especialidade oferecem maior valor hedônico que as realizadas em superlojas, as médias da escala VHC adaptada foram comparadas para os dois grupos por meio de um teste $t$. Não se observaram diferenças significativas (t(I92) $=0,065$, n.s.). Portanto, $\mathrm{H}_{3}$ deve ser rejeitada.

\section{DISCUSSÃO E CONCLUSÃO}

Uma vez que poucos estudos exploraram os efeitos de diferentes formatos de varejo sobre a produção de valor hedônico, o objetivo deste artigo era preencher parcialmente esse vazio na literatura ao comparar o valor hedônico derivado de compras feitas em diferentes tipos de lojas. De modo geral, a conclusão é que o valor hedônico não pode ser atribuído a classes específicas de varejistas, uma vez que, quando presentes, os efeitos foram pouco significativos.

Por exemplo, no primeiro estudo, ao contrário da hipótese, as compras feitas em lojas eletrônicas proporcionaram maior valor hedônico do que as realizadas em lojas tradicionais, embora essa diferença seja significativa apenas no nível de ıо\% de confiança. A fim de tentar identificar os motivos que poderiam ter conduzido a esses resultados, as razões expressas pelos respondentes por terem escolhido esta ou aquela loja para fazer a compra foram analisadas. 
Entre as pessoas que compraram em lojas tradicionais, as cinco principais razões mencionadas foram localização (23\%), preço (i9\%), disponibilidade de estoque (13\%), conveniência (I2\%) e hábito (9\%), razões tipicamente utilitárias e funcionais. É óbvio que não se esperava que os indivíduos mencionassem razões relativas a sentidos (iluminação, cheiros e cores), emoções ou sentimentos, particularmente porque se trata de razões inconscientes. A despeito disso, o número de pessoas que mencionaram apenas razões utilitárias para a sua escolha do varejista foi muito alto.

Entre as pessoas que compraram em lojas eletrônicas, um fato que chama a atenção é o número significativo (32\%) que mencionou confiança na loja como razão pela escolha do varejista. Uma das visões predominantes sobre a confiança é que ela envolve fortes componentes emocionais relacionados ao afeto (LEWIS; WEIGERT, I985), o que poderia parcialmente explicar o fato de que os consumidores que confiam numa loja eletrônica extraiam maior valor hedônico das compras feitas naquele varejista.

$\mathrm{Na}$ segunda pesquisa, constatou-se uma pequena associação entre o tamanho percebido de um shopping center e o valor hedônico de compras, mas o resultado foi significativo apenas no nível de ı०\%, e não se verificou nenhuma associação entre a variedade percebida de lojas de um shopping center e o valor hedônico. Esses resultados contradizem resultados anteriores que sugeriram esse tipo de relação utilizando medidas de percepção de tamanho de shopping center (KOWINSKI, I985; WAKEFIELD; BAKER, I998). Um teste adicional, desta vez não utilizando medidas de tamanho percebido, também não foi capaz de mostrar diferenças significativas de valor hedônico de compras feitas em shopping centers com diferentes tamanhos. É interessante notar que o shopping que proporcionou o menor valor hedônico de compras $(\mathrm{M}=3,49)$ tinha exatamente o mesmo número de lojas que aquele que proporcionou o maior valor hedônico $(M=3,89)$, e o maior shopping center da amostra proporcionou o segundo menor nível de valor hedônico $(\mathrm{M}=3,6 \mathrm{I})$. Esses resultados parecem indicar que o número de lojas de um shopping center não guarda nenhuma relação com o valor hedônico de compras.

$\mathrm{Na}$ última pesquisa, comparou-se o valor hedônico de compras feitas em lojas de especialidade e superlojas, e novamente não foram encontradas diferenças significativas. Embora as razões listadas pelos consumidores para justificar as suas escolhas das lojas em que fizeram compras terem fundamentalmente natureza utiliária e funcional (disponibilidade de estoque, 23\%; localização, $22 \%$; preços, $22 \%$ ), quando as razões declaradas para escolher lojas de especialidade e superlojas foram comparadas, algumas diferenças interessantes foram 
percebidas. Por exemplo, preço e conveniência parecem ser mais frequentemente mencionados por consumidores de superlojas, enquanto atendimento ao cliente, disponibilidade de estoque e avaliação global da loja ("gosto da loja") foram mais frequentemente mencionados por clientes de lojas de especialidades. Esse padrão de resultados sugere que os consumidores percebem algumas diferenças entre lojas de especialidade e superlojas, embora esse aspecto não tenha sido estabelecido quando se comparou o valor hedônico proporcionado pelos dois formatos.

Portanto, apesar de relevante, esse tipo de pesquisa, em que diferentes tipos de varejistas são comparados, não parece ser uma rota promissora de investigação. É mais provável que o valor hedônico origine-se em determinadas características da loja e na sua interação com determinados atributos do consumidor, como personalidade, inteligência e gênero (HOLBROOK, I986). A esse respeito, estudos futuros devem se concentrar em pesquisar características específicas dos varejistas e como elas podem interagir com características do consumidor na geração de valor hedônico.

Embora os resultados apresentados aqui não tenham sido muito encorajadores, é importante ressaltar as suas contribuições e as limitações impostas pelos desenhos de pesquisa adotados. Em relação às limitações, cabe ressaltar que é possível que o tamanho das amostras nas três pesquisas possa ter impedido a observação de resultados significativos, especialmente considerando que o método adotado foi o levantamento. Além do mais, a variabilidade dos dados causada pela grande variedade de varejistas analisados pode ter contribuído para eliminar efeitos significativos. É possível que resultados mais significativos tivessem sido obtidos caso as pesquisas tivessem se concentrado em um número menor de varejistas, tornando as amostras mais homogêneas.

Em relação às suas contribuições, deve-se salientar que a escala de valor hedônico foi testada em três pesquisas diferentes, e estabeleceram-se sua validade e confiabilidade. Dessa forma, futuros estudos em língua portuguesa sobre o valor hedônico de compras poderiam se utilizar da escala adaptada nessas três pesquisas.

O fato de os resultados não terem sido significativos parece ter deixado claro que essa linha de investigação não se mostrou muito esclarecedora. Mesmo estudos em que as hipóteses não se confirmam são importantes na ciência para aumentar o conhecimento em uma determinada área do conhecimento e para que futuros estudos levem em conta os resultados negativos obtidos anteriormente e aprimorem as hipóteses ou os métodos utilizados. 


\section{REFERÊENCIAS}

ARNOLD, M. J.; REYNOLDS, K. E. Hedonic shopping motivations. Journal of Retailing, v. 79, n. 2, p. $77-95,2003$.

BABIN, B. J.; ATTAWAY, J. S. Atmospheric affect as a tool for creating value and gaining share of customer. Journal of Business Research, v. 49, n. 2, p. 9I-99, 2000.

BABIN, B. J.; DARDEN, W. R. Consumer self-regulation in a retail environment. Journal of Retailing, v. 7I, n. I, p. 47-70, I995.

Good and bad shopping vibes: spending and patronage satisfaction. Journal of Business Research, v. 35, n. 3, p. 20I-206, I996.

BABIN, B. J.; DARDEN, W. R.; GRIFFIN, M. Work and/or fun: measuring hedonic and utilitarian shopping. Journal of Consumer Research, v. 20, n. 4, p. 644-656, I994.

BABIN, B. J. et al. Modeling consumer satisfaction and word-of-mouth: restaurant patronage in Korea. Journal of Service Marketing, v. I9, n. 3, p. I33-I39, 2005.

BARDHI, F.; ARNOULD, E. J. Thrift shopping: combining utilitarian thrift and hedonic benefits. Journal of Consumer Behavior, v. 4, n. 4, p. 223-233, 2005.

BATRA, R.; AHTOLA, O. T. Measuring the hedonic and utilitarian sources of consumer attitudes. Marketing Letters, v. 2, n. 2, p. I59-I70, I990.

BELLENGER, D. N.; KORGAONKAR, P. K. Profiling the recreational shopper. Journal of Retailing, v. 56, n. 3, p. $77-88$, I980.

BELLENGER, D. N.; ROBERTSON, D. H.; GREENBERG, B. A. Shopping center patronage motives. Journal of Retailing, v. 53, p. 29-38, I977.

BLOCH, P. H.; RIDGWAY, N.; NELSON, J. Leisure and the Shopping Mall. In: MONROE, K. B. (Ed.). Advances in consumer research. Ann Harbor: Association for Consumer Research, I99I. v. I8, p. $445-452$.

BURKE, R. Do you see what I see? The future of virtual shopping. Journal of the Academy of Marketing Science, v. 25, n. 4, p. 352-36I, I997.

CHILDERS, T. L.; CARR, C. L.; PECK, J. Hedonic and utilitarian motivations for online retail shopping behavior. Journal of Retailing, v. 77, n. 4, p. 5II-535, $200 \mathrm{I}$.

CHRISTIANSEN, T. et al. The effects of mall entertainment value on mall profitability. Journal of Shopping Center Research, v. 6, n. 2, p. 8-24, I999.

COTTET, P.; LICHTLÉ, M. C.; PLICHON, V. The role of value in services: a study in a retail environment. The Journal of Consumer Marketing, v. 23, n. 4, p. 219-227, 2006.

DEVELLIS, R. F. Scale development. Theory and applications. 2. ed. Thousand Oaks: Sage, 2003. DHAR, R.; WERTENBROCH, K. Consumer choice between hedonic and utilitarian goods. Journal of Marketing Research, v. 37, n. I, p. 60-7I, 2000.

DONOVAN, R. J.; ROSSITER, J. R. Store atmosphere: an environmental psychology approach. Journal of Retailing, v. 58, n. I, p. 34-57, I994.

DONOVAN, R. J. et al. Store atmosphere and purchasing behavior. Journal of Retailing, v. 70, n. 3, p. 283-294, I994.

EBIT. WebShoppers. I4. ed. Disponível em: <http://www.webshoppers.com.br/webshoppers.asp>. Acesso em: I5 dez. 2006. 
EROGLU, S. A.; MACHLEIT, K. A.; DAVIS, L. M. Empirical testing of a model of online store atmospherics and shopper responses. Psychology and Marketing, v. 20, p. I39-I50, 2003.

FINN, A.; LOUVIERE, J. J. Shopping center image, consideration, and choice: anchor store contribution. Journal of Business Research, v. 35, n. 3, p. 24I-25I, I996.

FIORE, A. M.; JIN, H.; KIM, J. For fun and profit: hedonic value from image interactivity and responses toward an online store. Psychology and Marketing, v. 22, n. 8, p. 669-694, 2005.

FIORE, A. M.; LEE, S. E.; KUNZ, G. I. Individual differences, motivations, and willingness to use mass customization options of fashion products. European Journal of Marketing, v. 38, n. 7 , p. 835-849, 2004 .

FISCHER, E.; ARNOLD, S. J. More than a labor of love: gender roles and Christmas gift shopping. Journal of Consumer Research, v. I7, n. 3, p. 333-445, I990.

HIRSCHMAN, E. C.; HOLBROOK, M. B. Hedonic consumption: emerging concepts, methods and propositions. Journal of Marketing, v. 46, p. 92-IOI, I982.

HOLBROOK, M. B. Aims, concepts, and methods for the representation of individual differences in esthetic responses to design features. Journal of Consumer Research, v. I3, n. 3, p. 337-347, I986. HOLBROOK, M. B.; HIRSCHMAN, E. C. The Experiential aspects of consumption: consumer fantasies, feelings, and fun. Journal of Consumer Research, v. 9, n. 2, p. I32-I40, I982.

JARVENPAA, S. L.; TODD, P. A. Consumer reactions to electronic shopping on the World Wide Web. International Journal of Electronic Commerce, v. I, p. 59-88, I997.

JONES, M. Entertaining shopping experience: an exploratory investigation. Journal of Retailing and Consumer Services, v. 6, p. 129-139, I999.

JONES, M. A.; REYNOLDS, K. E.; ARNOLD, M. J. Hedonic and utilitarian shopping value: investigating differential aspects on retail outcomes. Journal of Business Research, v. 59, n. 9, p. 974-98I, 2006.

KOTLER, P.; ARMSTRONG, G. Principles of marketing. Upper Saddle River: Prentice Hall, 200I. KOWINSKI, W. S. The malling of America. New York: William Morrow, I985.

LEWIS, J. D.; WEIGERT, A. Trust as a social reality. Social Forces, v. 64, n. 4, p. 967-985, 1985.

MANO, H.; OLIVER, R. L. Assessing the dimensionality and structure of the consumption experience: evaluation, feeling, and satisfaction. Journal of Consumer Research, v. 20, n. 3, p. 45I-466, I994.

MATTILA, A. S.; WIRTZ, J. Congruency of scent and music as a driver of in-store evaluations and behavior. Journal of Retailing, v. 77, n. 2, p. 273-289, 200 I.

NUNNALLY, J. C.; BERNSTEIN, I. H. Psychometric theory. 3. ed. New York: McGraw-Hill, I994. PAN, Y.; ZINKHAN, G. M. Determinants of retail patronage: a meta-analytical perspective. Journal of Retailing, v. 82, n. 3, p. 229-243, 2006.

PARK, C.; MOON, B. The relationship between product involvement and product knowledge: moderating roles of product type and product knowledge type. Psychology \& Marketing, v. 20, n. II, p. 977-997, 2003.

SHERMAN, F.; MATHUR, A.; SMITH, R. B. Store environment and consumer purchase behavior: mediating role of consumer emotions. Psychology and Marketing, v. I4, n. 4, p. 36I-378, I997. SHERRY JR., J. F. A sociocultural analysis of a midwestern American flea market. Journal of Consumer Research, v. I7, n. I, p. I3-30, I990. 
SHIM, S.; EASTLICK, M. A. The hierarchical influence of personal values on mall shopping attitude and behavior. Journal of Retailing, v. 74, n. I, p. I39-I6I, I998.

STOEL, L.; WICKLIFFE, V.; LEE, K. H. Attribute beliefs and spending as antecedents to shopping value. Journal of Business Research, v. 57, n. Iо, p. I067-I073, 2004.

STOLTMAN, J. J.; GENTRY, J. W.; ANGLIN, K. A. Shopping choices: the case of mall choice. In: HOLMAN, R; SOLOMON, M. (Ed.). Advances in consumer research. Provo: Association for Consumer Research, I99I. v. I8, p. 434-40.

VOSS, K. E.; SPANGENBERG, E. R.; GROHMANN, B. Measuring the hedonic and utilitarian dimensions of consumer attitude. Journal of Marketing Research, v. 40, n. 3, p. 310-320, 2003.

WAKEFIELD, K. L.; BAKER, J. Excitement at the mall: determinants and effects on shopping response. Journal of Retailing, v. 74, n. 4, p. 515-539, I998.

WAKEFIELD, K. L.; BARNES, J. H. Retailing hedonic consumption: a model of sales promotion of a leisure service. Journal of Retailing, v. 72, n. 4, p. 409-427, I996. 